American Journal of Applied Sciences 5 (4): 405-410, 2008

ISSN 1546-9239

(C) 2008 Science Publications

\title{
Cost Analysis of A System with Preventive Maintenance by Using the Kolmogorov's Forward Equations Method
}

\author{
Khaled Moh. El-Said \\ Department of Mathematics, Faculty of Science, Helwan University, Cairo, Egypt
}

\begin{abstract}
This study deals with cost analysis of a two- unit cold standby redundant system with preventive maintenance. The random failure occurs at random times which follow an exponential distribution and also the repair time are assumed to be exponentially distributed. Using the Kolmogorov's forward equations method. Several reliability characteristics are obtained. The mean time to system failure (MTSF) and the profit function are studied graphically.
\end{abstract}

Key words: Cost analysis, steady-state availability, mean time to system failure (MTSF), preventive maintenance (pm) and the kolmogorov's forward equations method

\section{INTRODUCTION}

Many authors ${ }^{[1,2]}$ have studied the two unit redundant systems with two types of repair. ${ }^{[3]}$ have studied stochastic analysis of a two - unit parallel system with partial and catastrophic failure and preventive maintenance. ${ }^{[4,5,6,7]}$ have studied the cost analysis of different systems. $\operatorname{In}^{[8]}$ by using the Kolmogorov's forward equations method. Evaluate the MTSF and availability of two different systems. This study devoted deals with cost analysis of a two- unit cold standby redundant system with two types of failure and preventive maintenance by using the Kolmogorov's forward equations. Initially one unit is operative and the other is kept as cold standby, i.e. it does not fail while standing by. Each unit works in two different types of failures. The both systems fail when both units fail totally. The failure and repair times are assumed to have exponential distribution. Using the special case study the effect of preventive maintenance on the system performance is shown by performing comparisons theoretically and graphically.

The following notations are adopted for the system:

$\alpha_{1}$ constant failure rate of type I.

$\alpha_{2}$ constant failure rate of type II.

$\beta_{1}$ constant repair rate of type I.

$\beta_{2}$ constant repair rate of type II.

$P_{i}(t)$ probability the system at time $t,(\mathrm{t} \geq 0)$ at state $\mathrm{S}_{\mathrm{i}}$. $\lambda$ constant rate for taking a unit into preventive maintenance.

$\delta$ constant rate end of preventive maintenance .

$\mathrm{O}$ the unit is operative.

$\mathrm{S}$ the unit is standby.

$F_{R 1}$ the failed unit is under repair of type $I$.

$F_{R 2}$ the failed unit is under repair of type II.

$\mathrm{F}_{\mathrm{W} 1}$ the failed unit is waited for repair of type $\mathrm{I}$.

$\mathrm{F}_{\mathrm{W} 2}$ the failed unit is waited for repair of type II.

$\mathrm{O}_{\mathrm{P}}$ the operative unit is under preventive maintenance.

$\mathrm{S}_{\mathrm{P}}$ the standby unit is under preventive maintenance.

The following system characteristics are studied:

i. Mean time to system failure.

ii. Steady state availability.

iii. Steady state busy period.

iv. Steady state, the expected frequency of preventive maintenance per unit time.

v. Cost analysis.

The following assumptions are adopted for the system:

1. The system consists of two similar units. Initially one unit is operative and the other unit is kept as cold standby.

2. Standby is switched to operative state in negligible time.

Corresponding Author: Khaled Moh. El-Said, Department of Mathematics, Faculty of Science, Helwan University, Cairo, Egypt 
3. A repaired unit works as a good as new.

4. The system is down when both units are nonoperative.

5. Each unit has two types of failure.

\section{Stochastic behavior of the system}

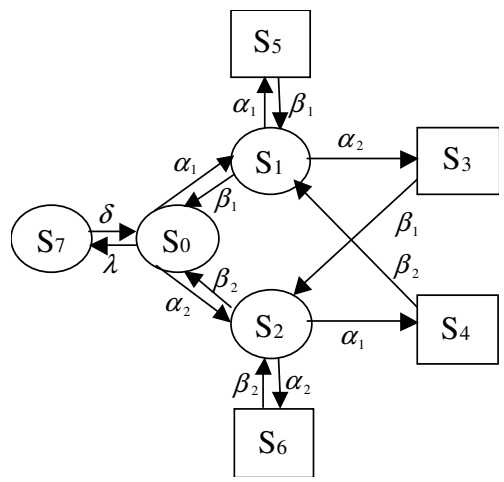

Up state

$\prod$ Down State

Fig. 1: Shows the states of the system

The system can take one of the following states:

$\mathrm{S}_{0}(\mathrm{O}, \mathrm{S}), \mathrm{S}_{1}\left(\mathrm{~F}_{\mathrm{R} 1}, \mathrm{O}\right) \mathrm{S}_{2}\left(\mathrm{~F}_{\mathrm{R} 2}, \mathrm{O}\right), \mathrm{S}_{3}\left(\mathrm{~F}_{\mathrm{R} 1}, \mathrm{~F}_{\mathrm{W} 2}\right)$,

$\mathrm{S}_{4}\left(\mathrm{~F}_{\mathrm{R} 2}, \mathrm{~F}_{\mathrm{W} 1}\right), \mathrm{S}_{5}\left(\mathrm{~F}_{\mathrm{R} 1}, \mathrm{~F}_{\mathrm{W} 1}\right), \mathrm{S}_{6}\left(\mathrm{~F}_{\mathrm{R} 2}, \mathrm{~F}_{\mathrm{W} 2}\right), \mathrm{S}_{7}\left(\mathrm{O}_{\mathrm{P}}, \mathrm{S}_{\mathrm{P}}\right)$.

3. Mean time to system failure: For Fig. 1 let $P_{i}(t)$

probability the system at time $t,(t \geq 0)$ at state $S_{i}$ let $\mathrm{P}(\mathrm{t})$ denote the probability row vector at time $\mathrm{t}$, then the initial conditions for this problem are

$\mathrm{P}(0)$

$=\left[\mathrm{P}_{0}(0), \mathrm{P}_{1}(0), \mathrm{P}_{2}(0), \mathrm{P}_{3}(0), \mathrm{P}_{4}(0), \mathrm{P}_{5}(0), \mathrm{P}_{6}(0), \mathrm{P}_{7}(0)\right]$

$=[1,0,0,0,0,0,0,0]$,

we obtain the following differential equation:

$\frac{\mathrm{dP}_{0}(\mathrm{t})}{\mathrm{dt}}=-\left(\alpha_{1}+\alpha_{2}+\lambda\right) \mathrm{P}_{0}(\mathrm{t})+\beta_{1} \mathrm{P}_{1}(\mathrm{t})+\beta_{2} \mathrm{P}_{2}(\mathrm{t})+\delta \mathrm{P}_{7}(\mathrm{t})$

$\frac{d \mathrm{P}_{1}(\mathrm{t})}{\mathrm{dt}}=-\left(\alpha_{1}+\alpha_{2}+\beta_{1}\right) \mathrm{P}_{1}(\mathrm{t})+\beta_{1} \mathrm{P}_{5}(\mathrm{t})+\alpha_{1} \mathrm{P}_{0}(\mathrm{t})+\beta_{1} \mathrm{P}_{4}(\mathrm{t})$

$\frac{d \mathrm{P}_{2}(\mathrm{t})}{\mathrm{dt}}=-\left(\alpha_{1}+\alpha_{2}+\beta_{2}\right) \mathrm{P}_{2}(\mathrm{t})+\beta_{2} \mathrm{P}_{6}(\mathrm{t})+\alpha_{2} \mathrm{P}_{0}(\mathrm{t})+\beta_{2} \mathrm{P}_{3}(\mathrm{t})$

$\frac{d P_{3}(t)}{d t}=-\beta_{2} P_{3}(t)+\alpha_{2} P_{1}(t)$

$\frac{\mathrm{dP}_{4}(\mathrm{t})}{\mathrm{dt}}=-\beta_{1} \mathrm{P}_{4}(\mathrm{t})+\alpha_{1} \mathrm{P}_{2}(\mathrm{t})$ $\frac{d P_{5}(t)}{d t}=-\beta_{1} P_{5}(t)+\alpha_{1} P_{1}(t)$

$\frac{\mathrm{dP}_{6}(\mathrm{t})}{\mathrm{dt}}=-\beta_{2} \mathrm{P}_{6}(\mathrm{t})+\alpha_{2} \mathrm{P}_{2}(\mathrm{t})$

$\frac{\mathrm{dP}_{7}(\mathrm{t})}{\mathrm{dt}}=-\delta \mathrm{P}_{7}(\mathrm{t})+\lambda \mathrm{P}_{0}(\mathrm{t})$

This can be written in the matrix form as

$\dot{\mathrm{P}}=\mathrm{QP}$

where,

$$
\mathrm{Q}=\left[\begin{array}{cccccccc}
-\left(\alpha_{1}+\alpha_{2}+\lambda\right) & \beta_{1} & \beta_{2} & 0 & 0 & 0 & 0 & \delta \\
\alpha_{1} & -\left(\alpha_{1}+\alpha_{2}+\beta_{1}\right) & 0 & 0 & \beta_{1} & \beta_{1} & 0 & 0 \\
\alpha_{2} & 0 & -\left(\alpha_{1}+\alpha_{2}+\beta_{2}\right) & \beta_{2} & 0 & 0 & \beta_{2} & 0 \\
0 & \alpha_{2} & 0 & -\beta_{2} & 0 & 0 & 0 & 0 \\
0 & 0 & \alpha_{1} & 0 & -\beta_{1} & 0 & 0 & 0 \\
0 & \alpha_{1} & 0 & 0 & 0 & -\beta_{1} & 0 & 0 \\
0 & 0 & \alpha_{2} & 0 & 0 & 0 & -\beta_{2} & 0 \\
\lambda & 0 & 0 & 0 & 0 & 0 & 0 & -\delta
\end{array}\right]
$$

to evaluate the transient solution is too complex therefore we will restrict ourselves in calculating the MTSF. To calculate the MTSF we take the transpose matrix of $\mathrm{Q}$ and delete the rows and columns for the absorbing state the new matrix is called A. the expected time to reach an absorbing state is calculated from

MTSF $=$

$$
\mathrm{E}\left[\mathrm{T}_{\mathrm{P}(0) \rightarrow \mathrm{P} \text { (absobing) }}\right]=\mathrm{P}(0)\left(-\mathrm{A}^{-1}\right)\left(\begin{array}{l}
1 \\
1 \\
1 \\
1
\end{array}\right)
$$

where,

$$
\begin{aligned}
& \mathrm{A}=\left[\begin{array}{cccc}
-\left(\alpha_{1}+\alpha_{2}+\lambda\right) & \alpha_{1} & \alpha_{2} & \lambda \\
\beta_{1} & -\left(\alpha_{1}+\alpha_{2}+\beta_{1}\right) & 0 & 0 \\
\beta_{2} & 0 & -\left(\alpha_{1}+\alpha_{2}+\beta_{2}\right) & 0 \\
\delta & 0 & 0 & -\delta
\end{array}\right] \\
& \text { MTSF }= \\
& \frac{\delta\left(2 \alpha_{1}+\alpha_{2}+\beta_{1}\right)\left(\alpha_{1}+\alpha_{2}+\beta_{2}\right)+\left(\alpha_{1}+\alpha_{2}+\beta_{1}\right)\left[\alpha_{2} \delta+\lambda\left(\alpha_{1}+\alpha_{2}+\beta_{2}\right)\right]}{\delta\left[\left(\alpha_{1}+\alpha_{2}\right)^{3}+\left(\alpha_{1}+\alpha_{2}\right)\left(\alpha_{1} \beta_{2}+\alpha_{2} \beta_{1}\right)\right]}
\end{aligned}
$$

4. Availability analysis: The initial conditions for this problem are the same as for the reliability case:

$\mathrm{P}(0)=[1,0,0,0,0,0,0,0]$,

the differential equations form can be expressed as:

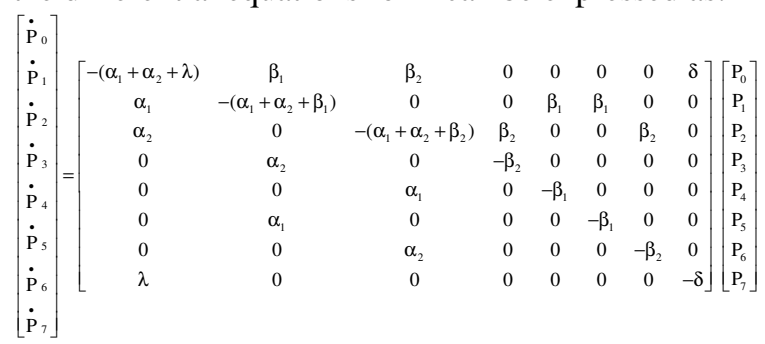


The steady state availability can be obtained using the following procedure. In the steady state, the derivatives of the state probabilities become zero. That allows us to calculate the steady state probabilities with. $\mathrm{A}(\infty)=\mathrm{P}_{0}(\infty)+\mathrm{P}_{1}(\infty)+\mathrm{P}_{2}(\infty)+\mathrm{P}_{7}(\infty)$

$\mathrm{QP}(\infty)=0$

or, in the matrix form

$$
\left[\begin{array}{cccccccc}
-\left(\alpha_{1}+\alpha_{2}+\lambda\right) & \beta_{1} & \beta_{2} & 0 & 0 & 0 & 0 & \delta \\
\alpha_{1} & -\left(\alpha_{1}+\alpha_{2}+\beta_{1}\right) & 0 & 0 & \beta_{1} & \beta_{1} & 0 & 0 \\
\alpha_{2} & 0 & -\left(\alpha_{1}+\alpha_{2}+\beta_{2}\right) & \beta_{2} & 0 & 0 & \beta_{2} & 0 \\
0 & \alpha_{2} & 0 & -\beta_{2} & 0 & 0 & 0 & 0 \\
0 & 0 & \alpha_{1} & 0 & -\beta_{1} & 0 & 0 & 0 \\
0 & \alpha_{1} & 0 & 0 & 0 & -\beta_{1} & 0 & 0 \\
0 & 0 & \alpha_{2} & 0 & 0 & 0 & -\beta_{2} & 0 \\
\lambda & 0 & 0 & 0 & 0 & 0 & 0 & -\delta
\end{array}\right]\left[\begin{array}{c}
P_{0} \\
P_{1} \\
P_{2} \\
P_{3} \\
P_{4} \\
P_{5} \\
P_{6} \\
P_{7}
\end{array}\right]=\left[\begin{array}{l}
0 \\
0 \\
0 \\
0 \\
0 \\
0 \\
0 \\
0
\end{array}\right]
$$

to obtain $\mathrm{P}_{0}(\infty), \mathrm{P}_{1}(\infty), \mathrm{P}_{2}(\infty), \mathrm{P}_{7}(\infty)$ we solve the equation (4.2) and the following normalizing condition:

$$
\begin{aligned}
& \mathrm{P}_{0}(\infty)+\mathrm{P}_{1}(\infty)+\mathrm{P}_{2}(\infty)+\mathrm{P}_{3}(\infty)+\mathrm{P}_{4}(\infty)+\mathrm{P}_{5}(\infty)+\mathrm{P}_{6}(\infty) \\
& +\mathrm{P}_{7}(\infty)=1
\end{aligned}
$$

We substitute the equation (4.3) in any one of the redundant rows in equation to (4.2) yield

$\left[\begin{array}{cccccccc}-\left(\alpha_{1}+\alpha_{2}+\lambda\right) & \beta_{1} & \beta_{2} & 0 & 0 & 0 & 0 & \delta \\ \alpha_{1} & -\left(\alpha_{1}+\alpha_{2}+\beta_{1}\right) & 0 & 0 & \beta_{1} & \beta_{1} & 0 & 0 \\ \alpha_{2} & 0 & -\left(\alpha_{1}+\alpha_{2}+\beta_{2}\right) & \beta_{2} & 0 & 0 & \beta_{2} & 0 \\ 0 & \alpha_{2} & 0 & -\beta_{2} & 0 & 0 & 0 & 0 \\ 0 & 0 & \alpha_{1} & 0 & -\beta_{1} & 0 & 0 & 0 \\ 0 & \alpha_{1} & 0 & 0 & 0 & -\beta_{1} & 0 & 0 \\ 0 & 0 & \alpha_{2} & 0 & 0 & 0 & -\beta_{2} & 0 \\ 1 & 1 & 1 & 1 & 1 & 1 & 1 & 1\end{array}\right]\left[\begin{array}{c}P_{0} \\ P_{1} \\ P_{2} \\ P_{3} \\ P_{4} \\ P_{5} \\ P_{6} \\ P_{7}\end{array}\right]=\left[\begin{array}{l}0 \\ 0 \\ 0 \\ 0 \\ 0 \\ 0 \\ 0 \\ 1\end{array}\right]$

The steady state availability $\mathrm{A}(\infty)$ is given by:

$$
\mathrm{A}(\infty)=\frac{\mathrm{N}_{1}}{\mathrm{D}_{1}}
$$

where,

$$
\begin{aligned}
\mathrm{N}_{1}= & \beta_{1} \beta_{2}\left[(\lambda+\delta)\left(\beta_{1} \beta_{2}+\alpha_{1} \beta_{1}+\alpha_{2} \beta_{2}\right)\right. \\
& \left.+\delta\left\{\alpha_{1}\left(\alpha_{1}+\alpha_{2}+\beta_{2}\right)+\alpha_{2}\left(\alpha_{1}+\alpha_{2}+\beta_{1}\right)\right\}\right], \\
\mathrm{D}_{1}= & \beta_{1} \beta_{2}(\lambda+\delta)\left(\beta_{1} \beta_{2}+\alpha_{1} \beta_{1}+\alpha_{2} \beta_{2}\right) \\
& +\delta \alpha_{2}^{2}\left[\beta_{2}\left(\alpha_{1}+\beta_{1}\right)+\beta_{1}\left(\alpha_{2}+2 \alpha_{1}+\beta_{1}\right)\right] \\
& +\delta \alpha_{1}^{2}\left[\beta_{1}^{2}+\alpha_{2} \beta_{1}+\alpha_{1} \beta_{2}+2 \alpha_{2} \beta_{2}+\beta_{1} \beta_{2}\right] \\
& +\delta \beta_{1} \beta_{2}\left[\alpha_{2} \beta_{1}+\alpha_{1} \beta_{2}+4 \alpha_{1} \alpha_{2}\right]
\end{aligned}
$$

Busy period analysis: The initial conditions for this problem are the same as for the reliability case:
$\mathrm{P}(0)$

$=\left[\mathrm{P}_{0}(0), \mathrm{P}_{1}(0), \mathrm{P}_{2}(0), \mathrm{P}_{3}(0), \mathrm{P}_{4}(0), \mathrm{P}_{5}(0), \mathrm{P}_{6}(0), \mathrm{P}_{7}(0)\right]$

$=[1,0,0,0,0,0,0,0]$,

the differential equations form can be expressed as:

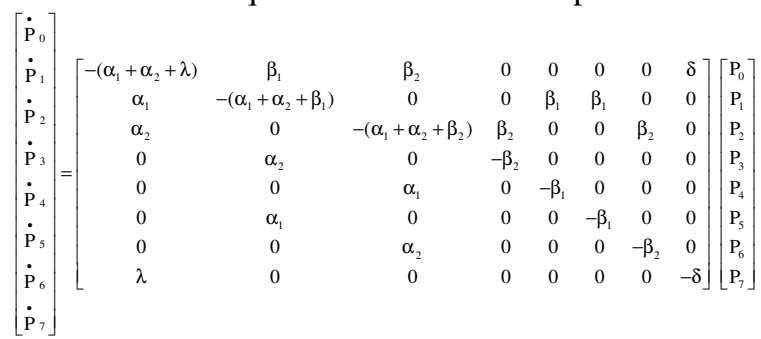

The steady state busy period can be obtained using the following procedure. In the steady state, the derivatives of the state probabilities become zero. That allows us to calculate the steady state probabilities with. $\mathrm{B}(\infty)=1-\left[\mathrm{P}_{0}(\infty)+\mathrm{P}_{7}(\infty)\right]$

$\mathrm{QP}(\infty)=0$

or, in the matrix form

$$
\left[\begin{array}{cccccccc}
-\left(\alpha_{1}+\alpha_{2}+\lambda\right) & \beta_{1} & \beta_{2} & 0 & 0 & 0 & 0 & \delta \\
\alpha_{1} & -\left(\alpha_{1}+\alpha_{2}+\beta_{1}\right) & 0 & 0 & \beta_{1} & \beta_{1} & 0 & 0 \\
\alpha_{2} & 0 & -\left(\alpha_{1}+\alpha_{2}+\beta_{2}\right) & \beta_{2} & 0 & 0 & \beta_{2} & 0 \\
0 & \alpha_{2} & 0 & -\beta_{2} & 0 & 0 & 0 & 0 \\
0 & 0 & \alpha_{1} & 0 & -\beta_{1} & 0 & 0 & 0 \\
0 & \alpha_{1} & 0 & 0 & 0 & -\beta_{1} & 0 & 0 \\
0 & 0 & \alpha_{2} & 0 & 0 & 0 & -\beta_{2} & 0 \\
\lambda & 0 & 0 & 0 & 0 & 0 & 0 & -\delta
\end{array}\right]\left[\begin{array}{c}
P_{0} \\
P_{1} \\
P_{2} \\
P_{3} \\
P_{4} \\
P_{5} \\
P_{6} \\
P_{7}
\end{array}\right]=\left[\begin{array}{l}
0 \\
0 \\
0 \\
0 \\
0 \\
0 \\
0 \\
0
\end{array}\right]
$$

to obtain $\mathrm{P}_{0}(\infty), \mathrm{P}_{7}(\infty)$,we solve the equation (5.2) and the following normalizing condition:

$$
\begin{aligned}
& \mathrm{P}_{0}(\infty)+\mathrm{P}_{1}(\infty)+\mathrm{P}_{2}(\infty)+\mathrm{P}_{3}(\infty)+\mathrm{P}_{4}(\infty)+\mathrm{P}_{5}(\infty)+\mathrm{P}_{6}(\infty) \\
& +\mathrm{P}_{7}(\infty)=1,
\end{aligned}
$$

we substitute the equation (5.3) in any one of the redundant rows in equation (5.2) to yield

$$
\left[\begin{array}{cccccccc}
-\left(\alpha_{1}+\alpha_{2}+\lambda\right) & \beta_{1} & \beta_{2} & 0 & 0 & 0 & 0 & \delta \\
\alpha_{1} & -\left(\alpha_{1}+\alpha_{2}+\beta_{1}\right) & 0 & 0 & \beta_{1} & \beta_{1} & 0 & 0 \\
\alpha_{2} & 0 & -\left(\alpha_{1}+\alpha_{2}+\beta_{2}\right) & \beta_{2} & 0 & 0 & \beta_{2} & 0 \\
0 & \alpha_{2} & 0 & -\beta_{2} & 0 & 0 & 0 & 0 \\
0 & 0 & \alpha_{1} & 0 & -\beta_{1} & 0 & 0 & 0 \\
0 & \alpha_{1} & 0 & 0 & 0 & -\beta_{1} & 0 & 0 \\
0 & 0 & \alpha_{2} & 0 & 0 & 0 & -\beta_{2} & 0 \\
1 & 1 & 1 & 1 & 1 & 1 & 1 & 1
\end{array}\right]\left[\begin{array}{l}
P_{0} \\
P_{1} \\
P_{2} \\
P_{3} \\
P_{4} \\
P_{5} \\
P_{6} \\
P_{7}
\end{array}\right]=\left[\begin{array}{l}
0 \\
0 \\
0 \\
0 \\
0 \\
0 \\
0 \\
1
\end{array}\right]
$$

The steady state busy period $\mathrm{B}(\infty)$ is given by

$\mathrm{B}(\infty)=1-\frac{\mathrm{N}_{2}}{\mathrm{D}_{1}}$

Where,

$\mathrm{N}_{2}=\beta_{1} \beta_{2}(\lambda+s)\left(\beta_{1} \beta_{2}+\alpha_{1} \beta_{1}+\alpha_{2} \beta_{2}\right)$ 
The expected frequency of preventive maintenance:

The initial conditions for this problem are the same as for the reliability case:

$\mathrm{P}(0)=[1,0,0,0,0,0,0,0]$,

the differential equations form can be expressed as:

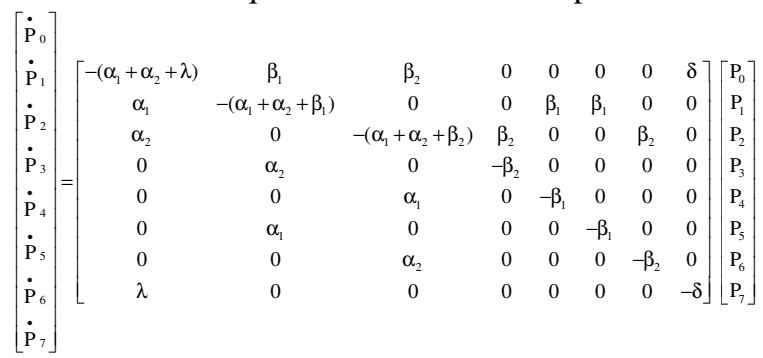

The steady state, the expected frequency of preventive maintenance per unit time can be obtained using the following procedure. In the steady state, the derivatives of the state probabilities become zero. That allows us to calculate the steady state probabilities with. $\mathrm{K}(\infty)=\mathrm{P}_{7}(\infty)$,

$$
\mathrm{QP}(\infty)=0 \text {, }
$$

or, in the matrix form

$$
\left[\begin{array}{cccccccc}
-\left(\alpha_{1}+\alpha_{2}+\lambda\right) & \beta_{1} & \beta_{2} & 0 & 0 & 0 & 0 & \delta \\
\alpha_{1} & -\left(\alpha_{1}+\alpha_{2}+\beta_{1}\right) & 0 & 0 & \beta_{1} & \beta_{1} & 0 & 0 \\
\alpha_{2} & 0 & -\left(\alpha_{1}+\alpha_{2}+\beta_{2}\right) & \beta_{2} & 0 & 0 & \beta_{2} & 0 \\
0 & \alpha_{2} & 0 & -\beta_{2} & 0 & 0 & 0 & 0 \\
0 & 0 & \alpha_{1} & 0 & -\beta_{1} & 0 & 0 & 0 \\
0 & \alpha_{1} & 0 & 0 & 0 & -\beta_{1} & 0 & 0 \\
0 & 0 & \alpha_{2} & 0 & 0 & 0 & -\beta_{2} & 0 \\
\lambda & 0 & 0 & 0 & 0 & 0 & 0 & -\delta
\end{array}\right]\left[\begin{array}{c}
P_{0} \\
P_{1} \\
P_{2} \\
P_{3} \\
P_{4} \\
P_{5} \\
P_{6} \\
P_{7}
\end{array}\right]=\left[\begin{array}{l}
0 \\
0 \\
0 \\
0 \\
0 \\
0 \\
0 \\
0
\end{array}\right]
$$

to obtain $\mathrm{P}_{7}(\infty)$ we solve the equation (6.2) and the following normalizing condition:

$\mathrm{P}_{0}(\infty)+\mathrm{P}_{1}(\infty)+\mathrm{P}_{2}(\infty)+\mathrm{P}_{3}(\infty)+\mathrm{P}_{4}(\infty)+\mathrm{P}_{5}(\infty)+\mathrm{P}_{6}(\infty)$ $+\mathrm{P}_{7}(\infty)=1$

We substitute the equation (6.3) in any one of the redundant rows in equation (6.2)to yield

$$
\left[\begin{array}{cccccccc}
-\left(\alpha_{1}+\alpha_{2}+\lambda\right) & \beta_{1} & \beta_{2} & 0 & 0 & 0 & 0 & \delta \\
\alpha_{1} & -\left(\alpha_{1}+\alpha_{2}+\beta_{1}\right) & 0 & 0 & \beta_{1} & \beta_{1} & 0 & 0 \\
\alpha_{2} & 0 & -\left(\alpha_{1}+\alpha_{2}+\beta_{2}\right) & \beta_{2} & 0 & 0 & \beta_{2} & 0 \\
0 & \alpha_{2} & 0 & -\beta_{2} & 0 & 0 & 0 & 0 \\
0 & 0 & \alpha_{1} & 0 & -\beta_{1} & 0 & 0 & 0 \\
0 & \alpha_{1} & 0 & 0 & 0 & -\beta_{1} & 0 & 0 \\
0 & 0 & \alpha_{2} & 0 & 0 & 0 & -\beta_{2} & 0 \\
1 & 1 & 1 & 1 & 1 & 1 & 1 & 1
\end{array}\right]\left[\begin{array}{c}
P_{0} \\
P_{1} \\
P_{2} \\
P_{3} \\
P_{4} \\
P_{5} \\
P_{6} \\
P_{7}
\end{array}\right]=\left[\begin{array}{l}
0 \\
0 \\
0 \\
0 \\
0 \\
0 \\
0 \\
1
\end{array}\right]
$$

The steady state, the expected frequency of preventive maintenance per unit time $\mathrm{K}(\infty)$ is given by
$K(\infty)=\frac{\lambda \beta_{1} \beta_{2}\left(\beta_{1} \beta_{2}+\alpha_{1} \beta_{1}+\alpha_{2} \beta_{2}\right)}{D_{1}}$

Cost analysis: The expected total profit per unit time incurred to the system in the steady-state is given by:

Profit $=$ total revenue - total cost

$$
\mathrm{PF}(\infty)=\mathrm{C}_{0} \mathrm{~A}(\infty)-\mathrm{C}_{1} \mathrm{~B}(\infty)-\mathrm{C}_{2} \mathrm{~K}(\infty)
$$

Where,

PF: is the profit incurred to the system,

$\mathrm{C}_{0}$ : is the revenue per unit up-time of the system,

$\mathrm{C}_{1}$ : is the cost per unit time which the system is under repair

$\mathrm{C}_{2}$ : is the cost per preventive maintenance.

Special case: After study the system when the preventive maintenance is not allowed, we get

The Mean Time to System Failure is given by

MTSF $=\frac{\left(\alpha_{1}+\alpha_{2}+\beta_{2}\right)\left(2 \alpha_{1}+\alpha_{2}+\beta_{1}\right)+\alpha_{2}\left(\alpha_{1}+\alpha_{2}+\beta_{1}\right)}{\left(\alpha_{1}+\alpha_{2}\right)^{3}+\left(\alpha_{1}+\alpha_{2}\right)\left(\alpha_{1} \beta_{2}+\alpha_{2} \beta_{1}\right)}$

The steady state availability is given by

$\hat{\mathrm{A}}(\infty)=\frac{\hat{\mathrm{N}}_{1}}{\hat{\mathrm{D}}_{1}}$

where,

$$
\begin{aligned}
\hat{\mathrm{N}}_{1}= & \beta_{1} \beta_{2}\left[\left(\beta_{1} \beta_{2}+\alpha_{1} \beta_{1}+\alpha_{2} \beta_{2}\right)+\alpha_{1}\left(\alpha_{1}+\alpha_{2}+\beta_{2}\right)\right. \\
& \left.+\alpha_{2}\left(\alpha_{1}+\alpha_{2}+\beta_{1}\right)\right] \\
\hat{\mathrm{D}}_{1}= & \beta_{1} \beta_{2}\left(\beta_{1} \beta_{2}+\alpha_{1} \beta_{1}+\alpha_{2} \beta_{2}\right) \\
& +\alpha_{2}^{2}\left[\beta_{2}\left(\alpha_{1}+\beta_{1}\right)+\beta_{1}\left(\alpha_{2}+2 \alpha_{1}+\beta_{1}\right)\right] \\
& +\alpha_{1}^{2}\left[\beta_{1}^{2}+\alpha_{2} \beta_{1}+\alpha_{1} \beta_{2}+2 \alpha_{2} \beta_{2}+\beta_{1} \beta_{2}\right] \\
& +\beta_{1} \beta_{2}\left[\alpha_{2} \beta_{1}+\alpha_{1} \beta_{2}+4 \alpha_{1} \alpha_{2}\right]
\end{aligned}
$$

The steady state busy period is given by

$$
\hat{\mathrm{B}}(\infty)=1-\frac{\hat{\mathrm{N}}_{2}}{\hat{\mathrm{D}}_{1}}
$$

Where,

$$
\hat{\mathrm{N}}_{2}=\beta_{1} \beta_{2}\left(\beta_{1} \beta_{2}+\alpha_{1} \beta_{1}+\alpha_{2} \beta_{2}\right)
$$

The steady state, the expected frequency of preventive maintenance per unit time is given by

$\hat{\mathrm{K}}(\infty)=\frac{\beta_{1} \beta_{2}\left(\beta_{1} \beta_{2}+\alpha_{1} \beta_{1}+\alpha_{2} \beta_{2}\right)}{\hat{\mathrm{D}}_{1}}$ 
Am. J. Appl. Sci. 5 (4): 405-410, 2008

Table 1:Relation between failure rate of type I and both the MTSF and the profit of the system (with and without PM)

\begin{tabular}{lllll}
\hline$\alpha_{1}$ & MTSF of the system with & $\begin{array}{l}\text { MTSF of the system } \\
\text { without PM }\end{array}$ & $\begin{array}{l}\text { The profit of the system } \\
\text { with PM }\end{array}$ & $\begin{array}{l}\text { The profit of the system } \\
\text { without PM }\end{array}$ \\
\hline 0.02 & PM & 49.01961 & 650.7418 & 573.1518 \\
0.03 & 81.37255 & 39.90148 & 588.5392 & 508.2898 \\
0.04 & 65.51724 & 33.56481 & 532.3624 & 452.9389 \\
0.05 & 54.62963 & 28.92282 & 482.1569 & 405.5684 \\
0.06 & 46.73452 & 25.38462 & 437.5162 & 364.8204 \\
0.07 & 40.76923 & 22.60308 & 397.8912 & 329.5545 \\
0.08 & 36.11525 & 20.36164 & 362.7034 & 298.8366 \\
0.09 & 32.38994 & 18.51852 & 331.4007 & 271.9087 \\
0.1 & 29.34473 & 16.97723 & 303.482 & 248.157 \\
\hline
\end{tabular}

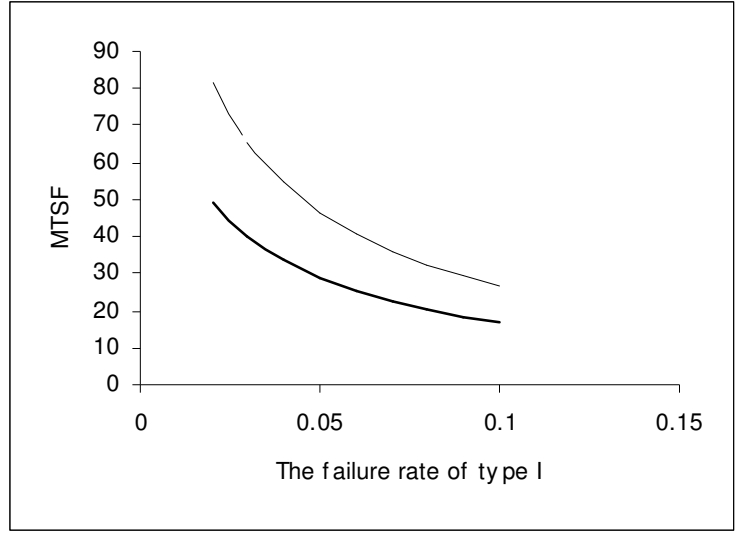

Fig. 2: Relation between the failure rate of type I and the MTSF

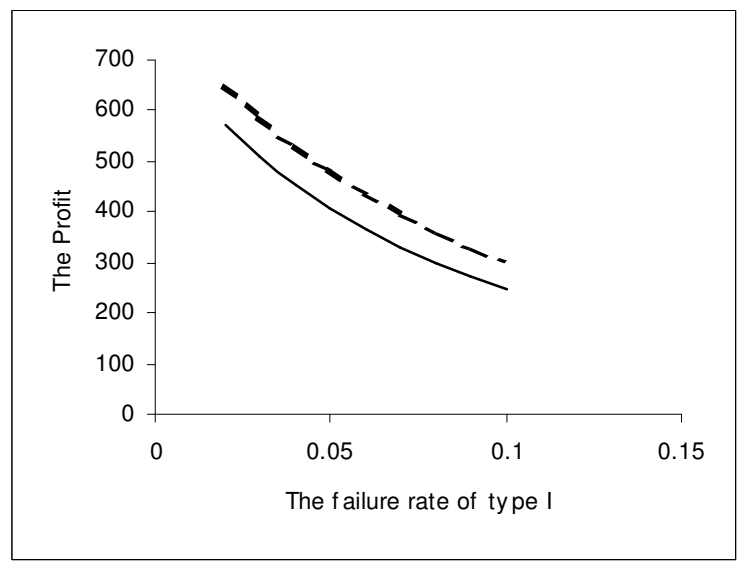

Fig. 3: Relation between the failure rate of type I and the Profit

The expected total profit per unit time incurred to the system in the steady-state is given by

$\mathrm{P} \hat{\mathrm{F}}(\infty)=\mathrm{C}_{0} \hat{\mathrm{A}}(\infty)-\mathrm{C}_{1} \hat{\mathrm{B}}(\infty)-\mathrm{C}_{2} \hat{\mathrm{K}}(\infty)$

\section{CONCLUSION}

By comparing the characteristic, MTSF and the profit function with respect to $\alpha_{1}$ for both systems with and without preventive maintenance graphically, it was observing that: -

The increase of failure rate $\alpha_{1}$ at constant $\alpha_{2}=0.04, \quad \beta_{1}=0.05, \beta_{2}=0.06, \lambda=0.02, \delta=0.02$, $\mathrm{C}_{0}=1000, \mathrm{C}_{1}=100, \mathrm{C}_{2}=100$. The MTSF and the profit function of the system decrease for both systems with and without preventive maintenance. We conclude that the system with preventive maintenance is more grater than the system without preventive maintenance with respect to the MTSF and the the profit function incurred to the model.

\section{REFERENCES}

1. Goel, L.R., G.C. Sharma and P. Gupta, 1986. Reliability analysis of a system with preventive maintenance inspection and two type of repair. Microelectron. Reliab., 26: 429-433.

2. Goel, L.R., R.Gupta and S.K. Singh, 1986. Profit analysis of a cold standby system with two repair distribution. Microelectron. Reliab., 25: 467-472.

3. Goel, L.R. and P. Gupta, 1984. stochastic analysis of a two-unit parallel system with partial and catastrophic failure and preventive maintenance. Microelectron. Reliab., 24: 881-883.

4. Gopalan, M.N. and H.E. Nagarwalia, 1985. Cost benefit analysis of a one server two-unit cold stand by system with repair and preventive maintenance. Microelectron. Relib., 25: 267-269.

5. Wang, K.H., C.H. Hsieh and C.H. Liou, 2006. Cost benefit analysis of series systems with cold standby components and a repairable service station. Qual. Technol. Quant. Manag., 3: 77-92. 
6. Said, El.,.M. Kh. and M.S. EL-Sherbeny, 2005. Evaluation of reliability and availability characteristics of two different systems by using linear first order differential equations. J. Math. Stat., 1: 119-123.

7. Said, El.,.M. Kh. and M.S. El-Sherbeny, 2005. Profit analysis of a two unit cold standby system with preventive maintenance and random change in units. J. Math. Stat., 1: 71-77.
8. Rander, M.C., K. Suresh and K. Ashok, 1994. Cost analysis of two dissimilar cold standby system with preventive maintenance and replacement of standby. Microelectron. Reliab., 34: 171-174. 